\title{
ERRATUM
}

In Issue 44/2 of Cereal Research Communications, on pp. 206-216, in the article:

\section{TaERECTA Responses to Phytohormones, $\mathrm{Mg}^{2+}$ Stress and Dehydration and Its Correlation with Stomatal Density in Bread Wheat}

Jia C. Zheng and Y.G. Hu; DOI: 10.1556/0806.44.2016.017

the affiliations of authors have not been indicated properly on title page.

The correct affiliations are as follows:

$$
\text { JIA C. ZHENG }{ }^{1,2} \text { and Y.G. Hu }{ }^{1,3 *}
$$

${ }^{1}$ State Key Laboratory of Crop Stress Biology in Arid Areas and College of Agronomy,

Northwest A\&F University, Yangling, Shaanxi, China, 712100

${ }^{2}$ College of Agronomy, Anhui Science and Technology University, Fengyang, Anhui, China, 233100

${ }^{3}$ Institute of Water Saving Agriculture in Arid Regions of China, Yangling, Shaanxi, China, 712100 